\title{
Una nueva clase de fármacos hipolipemiantes reduce la incidencia de infartos miocárdicos y cerebrales
}

\author{
A new class of lipid-lowering drugs reduces myocardial and stroke
}

Sabatine, Marc S. y col. N Engl J Med 2017; 376:1713-22

\begin{abstract}
Objetivos
Evaluar la eficacia clínica y la seguridad de evolocumab, cuando se agrega al tratamiento con estatinas de moderada o alta potencia, en pacientes con enfermedad cardiovascular (ECV) aterosclerótica clínicamente evidente.
\end{abstract}

\section{Diseño, lugar y pacientes}

Estudio aleatorizado, controlado con placebo, doble ciego, de grupos paralelos, multicentrico realizado en 49 países. Fueron incluidos 27.564 pacientes que habían sufrido un infarto de miocardio, accidente cerebrovascular (ACV) isquémico o tenían enfermedad vascular periférica sintomática y niveles de colesterol LDL menores a $70 \mathrm{mg} / \mathrm{dL}$ o de colesterol no HDL mayores a $100 \mathrm{mg} / \mathrm{dL}$. El $60 \%$ de los participantes fueron reclutados en Europa, 6,6 \% en Latinoamérica y aproximadamente $15 \%$ en Norteamérica, Asia y Sudáfrica. La duración promedio del seguimiento fue de 2,2 años.

\section{Intervención}

Los pacientes fueron asignados al azar en una proporción 1 a 1 a recibir evolocumab (140 mg subcutáneos cada dos semanas o $420 \mathrm{mg}$ subcutáneos una vez por mes, de acuerdo a su preferencia) o inyecciones de placebo. Como los dos esquemas de tratamiento tienen un efecto similar sobre el LDL, los participantes podían elegir cambiar cada 12 semanas. La asignación del tratamiento al azar se realizó con un sistema computarizado central estratificado por el nivel de LDL al final del tamizaje y por región.

\section{Medición de resultados principales}

El resultado principal fue compuesto (muerte cardiovascular; infarto de miocardio; accidente cerebrovascular, internación por angina inestable o revascularización coronaria). El resultado secundario clave incluyó los tres eventos cardiovasculares (ECV) más objetivos (muerte cardiovascular, infarto y ACV) ya que los criterios para internación por angina inestable o revascularización coronaria pueden variar. Todos los análisis de eficacia fueron realizados por intención de tratar. El tamaño de la muestra se basó en el resultado secundario clave y se estimó que se requerirían 1.630 de esos eventos para contar con un poder estadístico del $90 \%$ para detectar una reducción de riesgo relativo de $15 \%$ con evolocumab, comparado con placebo.

\section{Resultados principales}

Los resultados primarios se resumen en la tabla 1

Tabla 1. Efectividad del evolocumab para reducir los resultados de interés principal.

\begin{tabular}{c|c|c|c|c|c|}
\hline Resultado & Evolocumab & Placebo & RRR (IC95\%) & NNT & P \\
\hline Muerte cardiovascular, IAM, ACV & $9,8 \%$ & $11,3 \%$ & $15 \%$ (8 a 21) & 66 & $<0,001$ \\
\hline
\end{tabular}

IAM: infarto de miocardio; ACV: accidente cerebrovascular; RRR: reducción de riesgo relativo; IC95\%: intervalo de confianza del 95\%; NNT: número necesario a tratar.

En cuanto al resultado secundario, los eventos se presentaron en 816 pacientes en el grupo evolocumab $(5,9 \%)$ frente a 1013 pacientes del grupo placebo $(7,4 \%), \mathrm{HR}=0,8$ (IC $95 \% \quad 0,73$ a $0,88 ; p<0,001)$. Los resultados fueron consistentes entre los subgrupos.

\section{Conclusiones}

En este ensayo, el evolocumab (anticuerpo monoclonal que inhibe la proproteína convertasa subtilisina-kexin tipo 9-PCSK9), sumado a un tratamiento de base con estatina, disminuyó el nivel de colesterol LDL a una mediana de $30 \mathrm{mg} / \mathrm{dL}$ y redujo el riesgo de ECV. Estos hallazgos muestran que los pacientes con ECV aterosclerótica se benefician con reducciones del nivel de LDL por debajo de los objetivos actuales.

Fuente de financiamiento: El protocolo fue diseñado por el patrocinante (Amgen) y el Comité Ejecutivo. El grupo de estudio TIMI de investigación académica del Hospital Brigham and Women's de Boston tuvo completo acceso a la base de datos y generó independientemente sus análisis.

\section{Comentario}

Una pregunta importante para analizar el estudio que hemos resumido es: ¿en qué zona de la relación entre el nivel de colesterol LDL y el riesgo CV aterosclerótico tuvo lugar? Si bien esta relación es continua, no es lineal. Aunque nos han acostumbrado a representar los eventos en escala logarítmica, en cuyo caso la relación se ve lineal, esta es curvilínea y a valores séricos más bajos de LDL se obtienen menores beneficios absolutos para el mismo tratamiento hipolipemiante. Al Scandinavian Simvastatin Survival Study (4S), que redujo el LDL de aproximadamente 180 a $112 \mathrm{mg} /$ $\mathrm{dL}$ en 1312 pacientes con $20 \mathrm{mg}$ y a $127 \mathrm{mg} / \mathrm{dL}$ en 722 que requirieron $40 \mathrm{mg}$ de simvastatina, le bastaron 4.444 pacientes para mostrar reducción de la mortalidad total, mortalidad cardiovascular y eventos mayores, durante cinco años y medio de seguimiento ${ }^{1}$. En el caso de evolocumab solo era ético, en el momento del diseño del estudio, explorar valores de LDL inferiores a $55 \mathrm{mg} / \mathrm{dL}$ para verificar si se podían prevenir ECV. El descenso en valores absolutos fue similar al 4S (60 mg/dL) entre el tratamiento activo y placebo aunque, como era de esperar, el impacto clínico fue menor y solo para eventos no fatales. El seguimiento promedio fue de 2,2 años, con curvas de eventos continuamente divergentes. Un seguimiento más prolongado podría haber mostrado el beneficio clínico máximo de evolocumab.

\section{Conclusiones del comentador}

El beneficio para la salud pública de esta nueva opción terapéutica, que serviría para tratar personas con hipercolesterolemias familiares severas, a las verdaderamente intolerantes a estatinas o a quienes tienen escasa respuesta depende de su eficacia terapéutica y de la proporción de la población con indicación que pueda utilizarla, lo que nos obliga considerar su potencial impacto presupuestario en la toma de decisiones.

Gustavo Alcalá [ Centro Integral de Medicina de Alta Complejidad (CIMAC) San Juan; alcala.cardio@gmail.com ]

Alcalá G. Una nueva clase de fármacos hipolipemiantes reduce la incidencia de infartos miocárdicos y cerebrales. Evid Act Pract Ambul. $2018 ; 21$ (2): 50. Comentado de: Sabatine M y col. Evolocumab and Clinical Outcomes in Patients with Cardiovascular Disease. N Engl J Med 2017 ; $376: 1713-22$.

Referencias

1. Scandinavian Simvastatin Survival Study Group. Randomised trial of cholesterol lowering in 4444 patients with coronary heart disease: the Scandinavian Simvastatin Survival Study (4S). Lancet 1994;344: 1383-9. 